\title{
Controller for Three-phase Four-wire Shunt Active Power Filter by DC-bus Energy Regulation
}

\author{
R. Pindado ${ }^{1}$, P. Rodríguez ${ }^{2}$, J. Pou ${ }^{1}$ and I. Candela ${ }^{2}$ \\ ${ }^{1}$ Department of Electronics Engineering \\ ${ }^{2}$ Department of Electrical Engineering \\ ${ }^{1,2}$ Research Group on Power Quality and Renewable Energies (QuPER) \\ Technical University of Catalonia \\ Colón 1, 08222 Terrassa (Spain) \\ phone:+34 93 7398216, fax:+34 93 739601, e-mail: pindado@eel.upc.es
}

\begin{abstract}
This paper presents a three-phase four-wire shunt active power filter controlled under an energy approach. A nonconventional converter topology is presented and studied. With this topology, and considering harmonics and imbalances in utility voltage and load current, power developed by the active power filter is evaluated. From this evaluation, a controller based on the energy state of the dc-bus is designed. In this paper, an analytical study and verification by simulation are conducted.
\end{abstract}

\section{Key words}

Active Power Filter, Power Conditioning, DC-bus Energy Control.

\section{Introduction}

In three-phase four-wire shunt active power filter applications, two topologies for current-controlled voltage source inverter are commonly used, namely the four-leg full-bridge (FLFB) topology, and the three-leg split-capacitor (TLSC) topology. These topologies were presented at the beginning of the 90s [1], and numerous publications on their control have appeared ever since [2]-[4]. At first sight, the FLFB converter offers high control of its output voltages thanks to its greater number of legs. Nevertheless, interaction between the different legs makes necessary advanced current control techniques, such as space-vector modulation, in order to achieve suitable reference current tracking. The TLSC converter, having a smaller number of legs, permits each of the three legs to be controlled independently, making its current tracking control simpler than in the previous topology. However, in this last case the zero-sequence injected current flows through the dc-bus capacitors. This current gives rise to voltage sharing imbalance in the capacitors, and dc-bus voltage variation, which are undesirable effects in a precise control of the shunt active power filter (SAPF). Merging FLFB and TLSC topologies, an alternative four-leg split-capacitor (FLSC) topology, which solves the cited problems of the previous ones, can be obtained [5], see Fig. 1. In this topology,

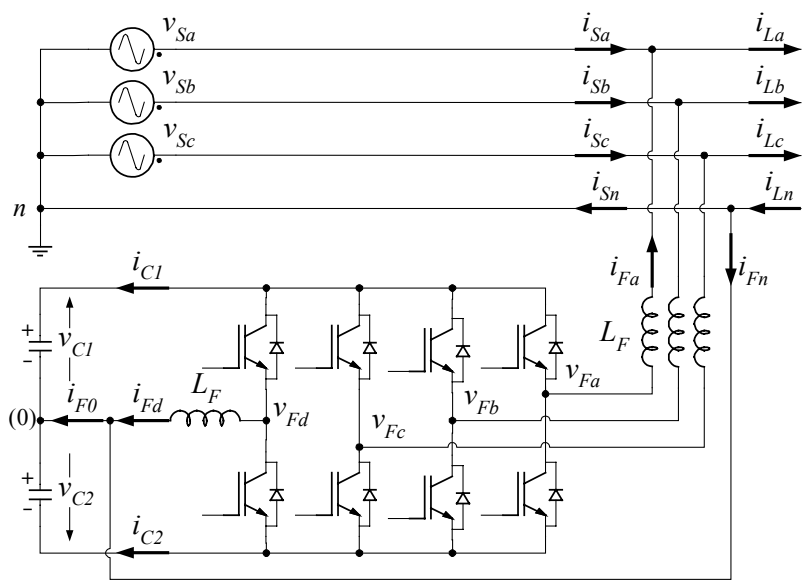

Fig. 1. Active power filter based in FLSC converter

each leg works independently from the other ones, and the current through leg $d$ can be regulated in order to achieve no current injected in the dc-bus midpoint; and therefore, dc-bus voltage imbalance can be cancelled.

\section{Averaged model of the FLSC topology}

In Fig. 1, if it is supposed that both dc-bus capacitors are equal, $C_{I}=C_{2}=C$, then each generic switching-leg can be represented by a state-space average model, in which the state equation is (1a) and the output equation is (1b).

$$
\begin{gathered}
{\left[\begin{array}{c}
\dot{i}_{F i} \\
\dot{v}_{C 1} \\
\dot{v}_{C 2}
\end{array}\right]=\left[\begin{array}{ccc}
0 & \frac{d_{i}}{L_{F i}} & \frac{\left(1-d_{i}\right)}{L_{F i}} \\
-\frac{d_{i}}{C} & 0 & 0 \\
-\frac{\left(1-d_{i}\right)}{C} & 0 & 0
\end{array}\right]\left[\begin{array}{l}
i_{F i} \\
v_{C 1} \\
v_{C 2}
\end{array}\right]+\left[\begin{array}{c}
-\frac{1}{L_{F i}} \\
0 \\
0
\end{array}\right] v_{S i}} \\
{\left[\begin{array}{l}
v_{F i} \\
i_{C 1} \\
i_{C 2}
\end{array}\right]=\left[\begin{array}{ccc}
0 & d_{i} & 1-d_{i} \\
-d_{i} & 0 & 0 \\
-\left(1-d_{i}\right) & 0 & 0
\end{array}\right]\left[\begin{array}{c}
i_{F i} \\
v_{C 1} \\
v_{C 2}
\end{array}\right]}
\end{gathered}
$$


In this averaged model, $d_{i} \in[0,1]$ is the duty cycle of the leg, where $i=\{a, b, c, d\}$. To simplify notation, variables in these equations represent averaged values over a switching period, that is: $i_{F i} \equiv \bar{i}_{F i}, v_{S i} \equiv \bar{v}_{S i}$, etc. From (1b), the duty cycle $d_{i}$ can be expressed as:

$$
d_{i}=\frac{\left(v_{F i}-v_{C 2}\right)}{v_{d c}},
$$

where $v_{d c}=v_{C 1}-v_{C 2}$ represents the dc-bus absolute voltage. Substituting (2) in current terms of (1b), and taking into account that the averaged value of the leg output voltage is $v_{F i}=v_{S i}+L_{F i} \dot{i}_{F i}$, then the currents in the capacitors are given by:

$$
\begin{gathered}
i_{C 1}=\frac{1}{v_{d c}}\left(v_{C 2} i_{F i}-v_{S i} i_{F i}-L_{F i} i_{F i} i_{F i}\right) \\
i_{C 2}=\frac{1}{v_{d c}}\left(-v_{C 1} i_{F i}+v_{S i} i_{F i}+L_{F i} i_{F i} i_{F i}\right)
\end{gathered}
$$

From (1), the averaged model of the active filter can be readily obtained by connecting four averaged switchinglegs and the rest of the circuit components. The four-wire averaged model can be depicted by means of the state equations (4).

$$
\dot{\mathbf{I}}_{F}=\frac{1}{L_{F}} \cdot\left(\mathbf{D} \cdot \mathbf{V}_{C}-\mathbf{V}_{S}\right) \quad ; \quad \dot{\mathbf{V}}_{C}=-\frac{1}{C} \cdot \mathbf{D}^{T} \cdot \mathbf{I}_{F},
$$

where $L_{F i}=L_{F} \forall i_{\mid i=\{a, b, c, d\}}, C_{I}=C_{2}=C$, and

$$
\begin{gathered}
\mathbf{I}_{F}=\left[\begin{array}{llll}
i_{F a} & i_{F b} & i_{F c} & i_{F d}
\end{array}\right]^{T} ; \quad \mathbf{V}_{C}=\left[\begin{array}{ll}
v_{C 1} & v_{C 2}
\end{array}\right]^{T} \\
\mathbf{V}_{S}=\left[\begin{array}{llll}
v_{S a} & v_{S b} & v_{S c} & 0
\end{array}\right]^{T} ; \quad \mathbf{D}=\left[\begin{array}{ll}
d_{a} & \left(1-d_{a}\right) \\
d_{b} & \left(1-d_{b}\right) \\
d_{c} & \left(1-d_{c}\right) \\
d_{d} & \left(1-d_{d}\right)
\end{array}\right] .
\end{gathered}
$$

Expression for current flowing through capacitors in FLSC topology will be obtained by summing terms shown in (3) for each leg, keeping in mind that $v_{S d}=0$. Resulting expressions are shown in (6), where $i_{F 0}$ is the injected current in the dc-bus midpoint, and $p_{F 3 \phi}$ is the instantaneous active power developed by the SAPF.

$$
\begin{gathered}
i_{C 1}=\frac{1}{v_{d c}}\left[v_{C 2} i_{F 0}-p_{F 3 \phi}-L_{F} \sum_{i=a, b, c, d}\left(i_{F i} \dot{i}_{F i}\right)\right] \\
i_{C 2}=\frac{1}{v_{d c}}\left[-v_{C 1} i_{F 0}+p_{F 3 \phi}+L_{F} \sum_{i=a, b, c, d}\left(i_{F i} \dot{i}_{F i}\right)\right]
\end{gathered}
$$

\section{DC-bus energy variation}

From (6), dc-bus energy variation can be expressed as:

$$
\begin{aligned}
& \Delta w_{d c}=w_{d c}-w_{d c}(0)=\int_{0}^{t}\left(v_{C 1} i_{C 1}+v_{C 2} i_{C 2}\right) d t \\
& =-\int_{0}^{t}\left(p_{F 3 \phi}+L_{F} \sum_{i=a, b, c, d} i_{F i} i_{F i}\right) d t=-\int_{0}^{t}\left(p_{F 3 \phi}+p_{L_{F}}\right) d t .
\end{aligned}
$$

Therefore, energy variation in the dc-bus only depends on the instantaneous active power developed by the filter, $p_{F 3 \phi}$, and on the instantaneous power associated to the tie inductances of the legs, $p_{L F}$. In a real implementation, additional power consumptions appear as a consequence of conduction and switching losses in the components of the APF. Considering these additional power losses, $p_{\text {loss }}$, (7) should be modified in order to obtain:

$$
\Delta w_{d c}=-\int_{0}^{t}\left(p_{F 3 \phi}+p_{\text {loss }}+p_{L_{F}}\right) d t .
$$

Expression (8), which is extremely simple, shows a linear relationship between energy variation in the dc-bus and power terms associated with the filter. Taking into account that SAPF purpose is to compensate for power oscillations in the load side, it seems logical that the SAPF control would be easily designed from the estimation of the dc-bus energy variation [6]. Therefore, from an energy approach, the SAPF could be represented by means of the block diagram shown in Fig. 2, where $p_{\text {int }}=p_{\text {loss }}+p_{L F}$. In Section 5, the controller dedicated to regulate energy variations in the dc-bus will be studied.

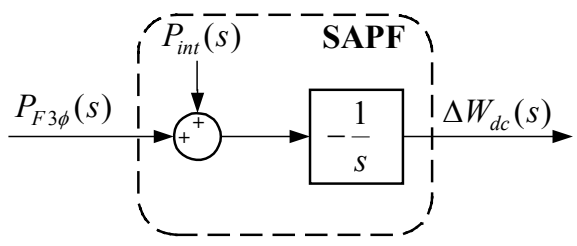

Fig. 2. Energy behavior of the SAPF

\section{Power developed by the SAPF}

Before designing the controller of the dc-bus energy variation, it is necessary to know the power developed by the SAPF when it is compensating for oscillations in the instantaneous power consumed by a generic load. Under completely general conditions, utility voltage might be unbalanced and distorted; thus, it can be expressed as:

$$
\begin{aligned}
& {\left[\begin{array}{c}
v_{L 0} \\
v_{L \alpha} \\
v_{L \beta}
\end{array}\right]=\left[\begin{array}{c}
v_{L 0} \\
0 \\
0
\end{array}\right]+\left[\begin{array}{c}
0 \\
v_{L \alpha}^{+} \\
v_{L \beta}^{+}
\end{array}\right]+\left[\begin{array}{c}
0 \\
v_{L \alpha}^{-} \\
v_{L \beta}^{-}
\end{array}\right]} \\
& \quad=\sum_{n=1}^{\infty} \sqrt{3}\left\{\sqrt{2} V_{o n}\left[\begin{array}{c}
\sin \left(\omega_{n} t+\phi_{o n}\right) \\
0 \\
0
\end{array}\right]\right. \\
& \left.+V_{+n}\left[\begin{array}{c}
\sin \left(\omega_{n} t+\phi_{+n}\right) \\
-\cos \left(\omega_{n} t+\phi_{+n}\right)
\end{array}\right]+V_{-n}\left[\begin{array}{c}
0 \\
\sin \left(\omega_{n} t+\phi_{-n}\right) \\
\cos \left(\omega_{n} t+\phi_{-n}\right)
\end{array}\right]\right\},
\end{aligned}
$$

where $V_{+n}, V_{-n}$ and $V_{o n}$ respectively correspond to the rms values of positive, negative and zero sequence voltage components of the $n$ order harmonic. In the same way, any generic load current might be expressed by means of (9), where $I_{+n}, I_{-n}$ and $I_{o n}$ again represent the RMS values of each current component. From (8) and (9) the mean value of the instantaneous active power consumed by the load is given by (10). 


$$
\begin{gathered}
{\left[\begin{array}{c}
i_{L 0} \\
i_{L \alpha} \\
i_{L \beta}
\end{array}\right]=\left[\begin{array}{c}
i_{L 0} \\
0 \\
0
\end{array}\right]+\left[\begin{array}{c}
0 \\
i_{L \alpha}^{+} \\
i_{L \beta}^{+}
\end{array}\right]+\left[\begin{array}{c}
0 \\
i_{L \alpha}^{-} \\
i_{L \beta}^{-}
\end{array}\right]} \\
=\sum_{n=1}^{\infty} \sqrt{3}\left\{\sqrt{2} I_{o n}\left[\begin{array}{c}
\sin \left(\omega_{n} t+\delta_{o n}\right) \\
0 \\
0
\end{array}\right]\right. \\
\left.+I_{+n}\left[\begin{array}{c}
\sin \left(\omega_{n} t+\delta_{+n}\right) \\
-\cos \left(\omega_{n} t+\delta_{+n}\right)
\end{array}\right]+I_{-n}\left[\begin{array}{l}
\sin \left(\omega_{n} t+\delta_{-n}\right) \\
\cos \left(\omega_{n} t+\delta_{-n}\right)
\end{array}\right]\right\} \\
\bar{p}_{L 3 \phi}=\sum_{n=1}^{\infty} 3\left[V_{+n} I_{+n} \cos \left(\phi_{+n}-\delta_{+n}\right)\right. \\
\left.+V_{-n} I_{-n} \cos \left(\phi_{-n}-\delta_{-n}\right)+V_{o n} I_{o n} \cos \left(\phi_{o n}-\delta_{o n}\right)\right]
\end{gathered}
$$

As in the previous section was indicated, the internal power losses of the SAPF represent additional power consumption. If this additional power is taken into account, the mean value of the instantaneous active power supplied by de source should be:

$$
\bar{p}_{S 3 \phi}=\bar{p}_{L}+\bar{p}_{L 0}+\bar{p}_{i n t}=\bar{p}_{L 3 \phi}+\bar{p}_{i n t} .
$$

In (11), the power term corresponding to the internal power of the SAPF, $\bar{p}_{\text {int }}$, might represent not only the power losses of the SAPF components, but also any additional load intentionally connected to the dc-bus, e.g. a voltage source inverter coupled to the dc-bus in order to drive an induction motor. In this back-to-back topology, the source side converter should act as a PWM boost rectifier, which additionally implements the active filtering capability.

If it is imposed as a condition that currents drawn from the source should be balanced, sinusoidal at fundamental frequency and in phase with the positive sequence voltage of the utility, then this currents might be expressed as:

$$
\begin{aligned}
\mathbf{I}_{S} & =\left[\begin{array}{c}
i_{S 0} \\
i_{S \alpha} \\
i_{S \beta}
\end{array}\right]=\left[\begin{array}{c}
0 \\
i_{S \alpha}^{+1} \\
i_{S \beta}^{+1}
\end{array}\right]=\left[P \rightarrow I_{\alpha \beta}^{+1}\right]\left[\begin{array}{c}
0 \\
\bar{p}_{S 3 \phi} \\
0
\end{array}\right] \\
& =\frac{\bar{p}_{S 3 \phi}}{\sqrt{3} V_{+1}}\left[\begin{array}{c}
0 \\
\sin \left(\omega t+\phi_{+1}\right) \\
-\cos \left(\omega t+\phi_{+1}\right)
\end{array}\right]
\end{aligned}
$$

where

$$
\left[P \rightarrow I_{\alpha \beta}^{+1}\right]=\frac{1}{\left(v_{L \alpha}^{+1}\right)^{2}+\left(v_{L \beta}^{+1}\right)^{2}}\left[\begin{array}{ccc}
0 & 0 & 0 \\
0 & v_{L \alpha}^{+1} & -v_{L \beta}^{+1} \\
0 & v_{L \beta}^{+1} & v_{L \alpha}^{+1}
\end{array}\right]
$$

In (13), $v_{L \alpha}^{+1}$ and $v_{L \beta}^{+1}$ correspond to the positive sequence utility voltage at fundamental frequency, which should be obtained by means of a synchronous double reference frame PLL (SDRF-PLL) [7]-[8].

If the SAPF achieves that currents flowing into the source side agree with (12), then the instantaneous powers supplied by the source would be:

$$
\begin{aligned}
& {\left[\begin{array}{c}
p_{S 0} \\
p_{S} \\
q_{S}
\end{array}\right]=\left[I_{\alpha \beta 0} \rightarrow P\right]\left[\begin{array}{c}
0 \\
i_{S \alpha}^{+1} \\
i_{S \beta}^{+1}
\end{array}\right]=\left[\begin{array}{c}
0 \\
\bar{p}_{S} \\
0
\end{array}\right]+\left[\begin{array}{c}
0 \\
\widetilde{p}_{S} \\
\widetilde{q}_{S}
\end{array}\right]=\left[\begin{array}{c}
0 \\
\bar{p}_{S 3 \phi} \\
0
\end{array}\right]} \\
& +\frac{\bar{p}_{S 3 \phi}}{V_{+1}}\left\{\left[\begin{array}{c}
0 \\
\sum_{n=2}^{\infty} V_{+n} \cos \left((n-1) \omega_{1} t+\phi_{+n}-\phi_{+1}\right) \\
\sum_{n=2}^{\infty}-V_{+n} \sin \left((n-1) \omega_{1} t+\phi_{+n}-\phi_{+1}\right)
\end{array}\right]\right. \\
& \left.-\left[\begin{array}{c}
0 \\
\sum_{n=1}^{\infty} V_{-n} \cos \left((n+1) \omega_{1} t+\phi_{-n}+\phi_{+1}\right) \\
\sum_{n=1}^{\infty} V_{-n} \sin \left((n+1) \omega_{1} t+\phi_{-n}+\phi_{+1}\right)
\end{array}\right]\right\},
\end{aligned}
$$

where

$$
\left[I_{\alpha \beta 0} \rightarrow P\right]=\left[\begin{array}{ccc}
v_{L 0} & 0 & 0 \\
0 & v_{L \alpha} & v_{L \beta} \\
0 & v_{L \beta} & -v_{L \alpha}
\end{array}\right]
$$

In (14), oscillatory terms in real and imaginary powers appear as a consequence of interaction between positive sequence sinusoidal current and all those voltage source components with a different frequency and/or sequence. Subtracting instantaneous powers supplied by the source from instantaneous powers consumed by the load, the instantaneous powers developed by the SAPF are:

$$
\begin{aligned}
{\left[\begin{array}{c}
p_{F 0} \\
p_{F} \\
q_{F}
\end{array}\right] } & =\left[\begin{array}{c}
\bar{p}_{F 0} \\
\bar{p}_{F} \\
\bar{q}_{F}
\end{array}\right]+\left[\begin{array}{c}
\tilde{p}_{F 0} \\
\tilde{p}_{F} \\
\tilde{q}_{F}
\end{array}\right]=\left[\begin{array}{c}
p_{L 0} \\
p_{L} \\
q_{L}
\end{array}\right]-\left[\begin{array}{c}
p_{S 0} \\
p_{S} \\
q_{S}
\end{array}\right] \\
& \left.=\left\{\left[\begin{array}{c}
\bar{p}_{L 0} \\
\bar{p}_{L} \\
\bar{q}_{L}
\end{array}\right]+\left[\begin{array}{c}
\tilde{p}_{L 0} \\
\tilde{p}_{L} \\
\widetilde{q}_{L}
\end{array}\right]\right\}-\left\{\begin{array}{c}
0 \\
\bar{p}_{L}+\bar{p}_{L 0}+\bar{p}_{i n t} \\
0
\end{array}\right]+\left[\begin{array}{c}
0 \\
\tilde{p}_{S} \\
\widetilde{q}_{S}
\end{array}\right]\right\} \\
& =\left[\begin{array}{c}
\bar{p}_{L 0} \\
-\bar{p}_{L 0}-\bar{p}_{i n t} \\
\bar{q}_{L}
\end{array}\right]+\left[\begin{array}{c}
\tilde{p}_{L 0} \\
\tilde{p}_{L}-\tilde{p}_{S} \\
\tilde{q}_{L}-\tilde{q}_{S}
\end{array}\right] .
\end{aligned}
$$

As a conclusion, the instantaneous active and imaginary powers developed by the SAPF are given by:

$$
\begin{gathered}
p_{F 3 \phi}=\widetilde{p}_{L 3 \phi}-\widetilde{p}_{S 3 \phi}-\bar{p}_{i n t}, \\
q_{F}=\bar{q}_{L}+\widetilde{q}_{L}-\widetilde{q}_{S} .
\end{gathered}
$$

Therefore, speaking of instantaneous active power, the SAPF is supplying the load power oscillations, absorbing the source power oscillations, and absorbing the necessary power to compensate its internal power consumptions.

From a conventional approach, the SAPF control might be implemented by means of the diagram shown in Fig. $3 \mathrm{a}$. In this figure, the cut-off frequency of the low-pass 
(a)

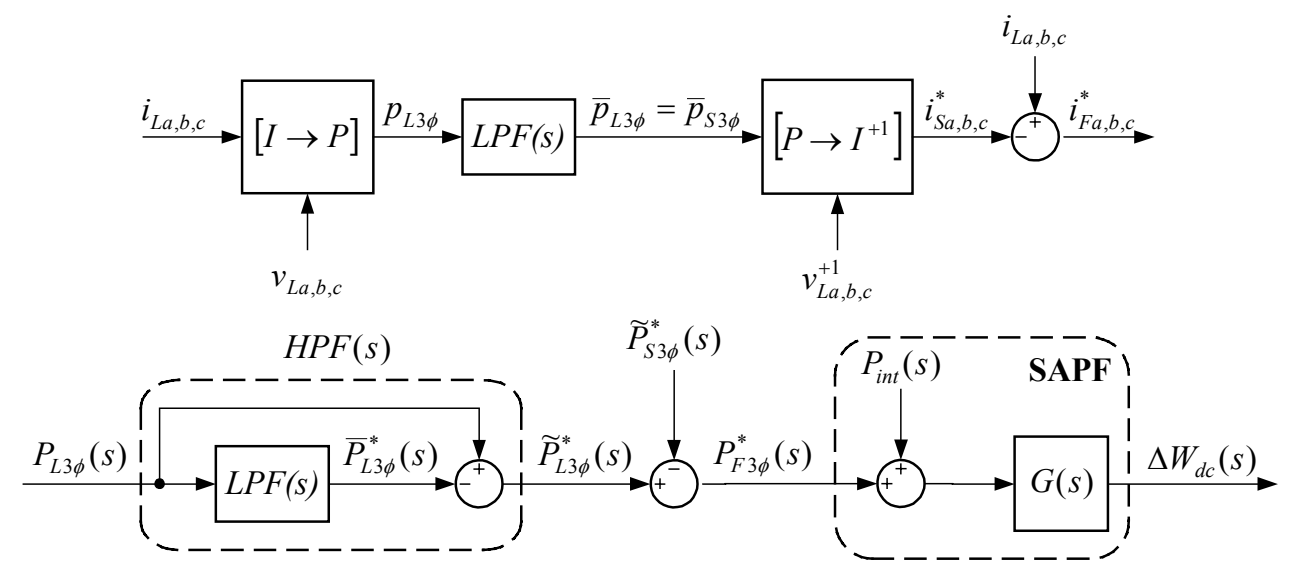

Fig. 3. Conventional SAPF control based on instantaneous active power calculation in the load

filter should be selected taking into account that the minimum frequency of oscillations in instantaneous active power consumed by the load can become equal to $2 \omega_{S}$, being $\omega_{S}$ the fundamental utility frequency. Therefore, the transfer function of this low-pass filter is:

$$
\operatorname{LPF}(s)=\frac{\omega_{f}^{2}}{\left(s+\omega_{f}\right)^{2}} \quad ; \quad \omega_{f}=\frac{2 \omega_{S}}{10} .
$$

Taking into account that:

$$
\begin{aligned}
& i_{S a, b, c}^{*} \cdot v_{L a, b, c}=\bar{p}_{S 3 \phi}^{*}+\widetilde{p}_{S 3 \phi}^{*}=\bar{p}_{L 3 \phi}^{*}+\widetilde{p}_{S 3 \phi}^{*}, \\
& i_{L a, b, c} \cdot v_{L a, b, c}=\bar{p}_{L 3 \phi}+\widetilde{p}_{L 3 \phi}, \\
& i_{F a, b, c}^{*} \cdot v_{L a, b, c}=p_{F 3 \phi}^{*},
\end{aligned}
$$

the control diagram of Fig. 3a gives rise to the power flow diagram shown in Fig. 3b. In this last diagram, the SAPF energy model has been added in order to describe the energy behavior of the dc-bus. If it is kept in mind that $G(s)=-s^{-1}$ and

$$
H P F(s)=(1-\operatorname{LPF}(s))=\frac{s\left(s+2 \omega_{f}\right)}{\left(s+\omega_{f}\right)^{2}},
$$

the analysis of the diagram shown in Fig. $3 b$ conducts to the following expression for the dc-bus energy variation:

$$
\Delta W_{d c}(s)=G(s)\left[\operatorname{HPF}(s) \cdot P_{L 3 \phi}(s)+P_{i n t}(s)-\widetilde{P}_{S 3 \phi}^{*}(s)\right] \cdot
$$

Transfer function shown in (21) shows as internal power consumptions of the SAPF are not compensated, which is enough reason to justify the necessity of a control loop modification. However, in order to properly establish the control loop modifications, the system will be firstly analyzed considering that $P_{i n t}(s)-\widetilde{P}_{S 3 \phi}^{*}(s)=0$. Under this condition, the transfer function that relates dc-bus energy variation and instantaneous active power of the load is:

$$
\frac{\Delta W_{d c}}{P_{L 3 \phi}}(s)=G(s) H P F(s)=-\frac{s+2 \omega_{f}}{\left(s+\omega_{f}\right)^{2}} .
$$

In (22), the steady state value of step response is $\Delta w_{d c-s s}=-2 / \omega_{f}$. Elimination of this steady state error in the dc-bus energy variation justifies further the need of a suitable energy controller.

\section{Controller of the dc-bus energy variation}

The first version of the proposed controller of the dc-bus energy variation is shown in Fig. 4. In this figure, $C(s)$ is a proportional controller with a gain equal to $k$, and $H(s)$ is constituted by a notch filter in cascade with a low-pass filter tuned at $\omega_{h}=2 \omega_{S}$. Its transfer function is:

$$
H(s)=\frac{\omega_{h}^{2}\left(s^{2}+\omega_{h}^{2}\right)}{\left(s^{2}+2 \xi_{h} \omega_{h} s+\omega_{h}^{2}\right)^{2}} \quad ; \quad \omega_{h}=2 \cdot \omega_{S}, \xi_{h}=1
$$

Characteristic transfer functions of the proposed controller are shown in (24).

$$
\begin{aligned}
& \frac{P_{F 3 \phi}^{*}}{P_{L 3 \phi}}(s)= \frac{H P F(s)}{1-G(s) C(s) H(s)} \\
&= \frac{s^{2}\left(s+2 \omega_{f}\right)}{\left(1+\frac{k \omega_{h}^{2}\left(s^{2}+\omega_{h}^{2}\right)}{s\left(s^{2}+2 \xi_{h} \omega_{h} s+\omega_{h}^{2}\right)^{2}}\right)\left(s+\omega_{f}\right)^{2}} \\
& \frac{\Delta W_{d c}}{P_{L 3 \phi}}(s)=G(s) \frac{P_{F 3 \phi}^{*}}{P_{L 3 \phi}}(s)
\end{aligned}
$$

Since the whole oscillatory active power of the load at $\omega=2 \omega_{S}$ should be developed by the SAPF, transfer function shown in (24a) should present unitary gain at this frequency. Thereby, proportional gain is limited to $k=\omega_{\text {f. }}$. With this value for $k$, and supposing that $H(s)$ block has not been included in the controller, the active power consumed by the load at $\omega=2 \omega_{S}$ would be slightly lagged respect to the active power supplied by the SAPF. Hence, an incorrect compensation of this load power

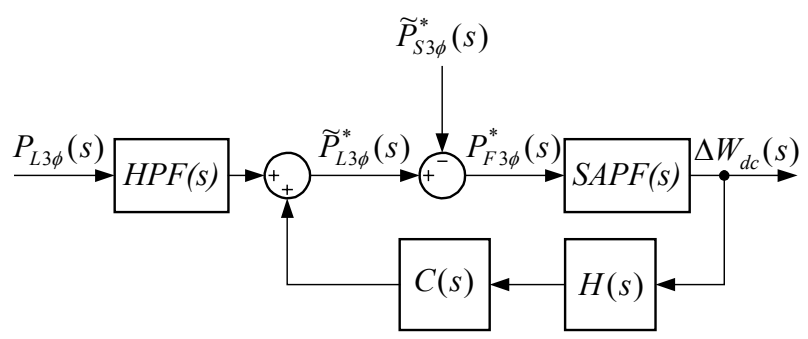

Fig. 4. Initial controller of dc-bus energy variation 
component would be done by the SAPF. To avoid this problem, the $H(s)$ block is added to the control loop. Fig. 5 shows temporal evolution of dc-bus energy variation when a unitary step appears in the instantaneous active power consumed by the load. For this plot, $\omega_{S}=2 \cdot \pi \cdot 50$ $\mathrm{rad} / \mathrm{s}, k=\omega_{f}=2 \cdot \pi \cdot 10 \mathrm{~W} / \mathrm{J}, \omega_{h}=2 \cdot \pi \cdot 100 \mathrm{rad} / \mathrm{s}$ and $\xi_{f}=\xi_{h}=1$ have been considered.

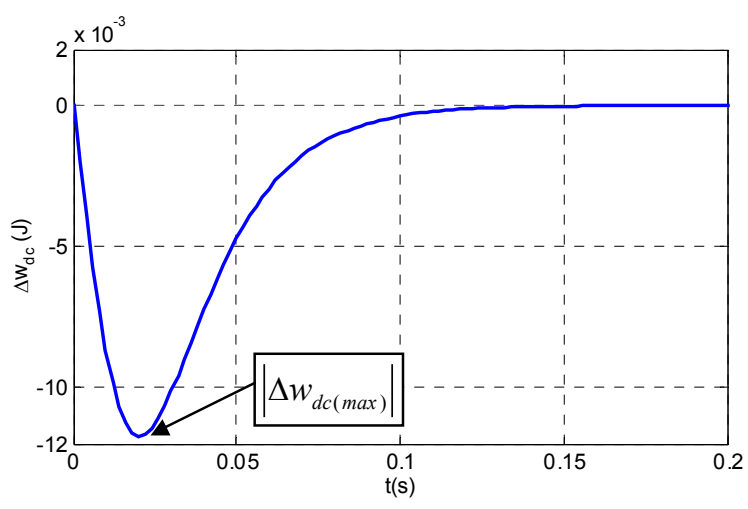

Fig. 5. Step response of the closed-loop system

Analytical study of (24b) permits to obtain a very accurate approximate expression for the maximum absolute value of the waveform plotted in Fig. 5. This expression is shown in (25), where $\left|\Delta P_{L}\right|$ is the absolute value of the load power step.

$$
\left|\Delta w_{d c(\max )}\right|=\frac{\left|\Delta P_{L}\right|}{k\left(1-2 \xi_{h} \frac{\omega_{f}}{\omega_{h}}\right)}(1+\sqrt{2}) e^{-\sqrt{2}}
$$

If it is supposed that current injected by the converter leg $d$ is properly controlled in order to achieve balance in the dc-bus voltage sharing [9]-[10], then energy stored in the dc-bus can be calculated by:

$$
w_{d c}=\frac{1}{4} C\left(v_{C 1}-v_{C 2}\right)^{2}=\frac{1}{4} C v_{d c}^{2},
$$

where $v_{d c}$ is the dc-bus absolute voltage and $C_{1}=C_{2}=C$. Therefore, dc-bus energy variation regarding an initial value adopted as reference would be:

$$
\Delta w_{d c}=\frac{1}{4} C\left(v_{d c}^{2}-v_{d c(r e f)}^{2}\right)
$$

where $v_{d c(r e f)}$ is the dc-bus voltage adopted as a reference. From (25) and (27), it is possible to determinate the proper value for the dc-bus capacitors in order to ensure that dc-bus absolute voltage does not overpass a limit value, $v_{d c(l i m)}$, when a certain load power step appears.

$$
C \geq \frac{4 \cdot\left|\Delta w_{d c(\max )}\right|}{\left|v_{d c(\text { ref })}^{2}-v_{d c(\text { lim })}^{2}\right|}
$$

So far, it has been considered that $P_{\text {int }}(s)-\widetilde{P}_{S 3 \phi}^{*}(s)=0$. If now these power terms are kept in mind, an analysis of the diagram shown in Fig. 4 allows obtaining the expression of (29) for dc-bus energy variation.

$$
\begin{aligned}
\Delta W_{d c}(s) & =\frac{G(s)}{1-G(s) C(s) H(s)}\left[\operatorname{HPF}(s) P_{L 3 \phi}(s)\right. \\
& \left.+\left(P_{\text {int }}(s)-\widetilde{P}_{S 3 \phi}^{*}(s)\right)\right]
\end{aligned}
$$

In (29), no all of the power terms affect in the same way over the dc-bus energy variation. Taking into account that internal power consumptions can become important when additional loads are connected to the dc-bus, it should be interesting to have a unique transfer function that univocally relates all of the power terms with the dcbus energy variation. To achieve this goal, a second control loop is added to the initial controller. Fig. 6 shows the new structure of the dc-bus energy variation controller. In this diagram, real structure of the high-pass filter has been considered, $D(s)=s$ is a derivative block, and $L P F(s)$ is the low-pass filter shown in (18). Besides, a new power magnitude has been defined, $P_{F(\text { eff })}(s)$, which corresponds to the effective power that really modifies the energy stored in the dc-bus. Analyzing the diagram shown in Fig. 6, the following transfer function is obtained:

$$
\frac{\Delta W_{d c}}{P_{L 3 \phi}+P_{\text {int }}-\widetilde{P}_{S 3 \phi}^{*}}(s)=\operatorname{HPF}(s) \frac{G(s)}{1-G(s) C(s) H(s)} .
$$

Expression of (30) denotes that dc-bus energy variation is completely controlled, following an identical evolution versus variations in any of the powers that affect to the energy state of the dc-bus. Analyzing control diagram shown in Fig. 6 is obtained that:

$$
\begin{aligned}
\frac{\bar{P}_{L 3 \phi}^{*}(s)}{\Delta W_{d c}(s)} & =-\frac{H(s) C(s)+D(s) L P F(s)}{1-L P F(s)} \\
& =-\left[H(s) C(s) F_{1}(s)+F_{2}(s)\right],
\end{aligned}
$$

where $F_{1}(s)$ and $F_{2}(s)$ are defined in (32).

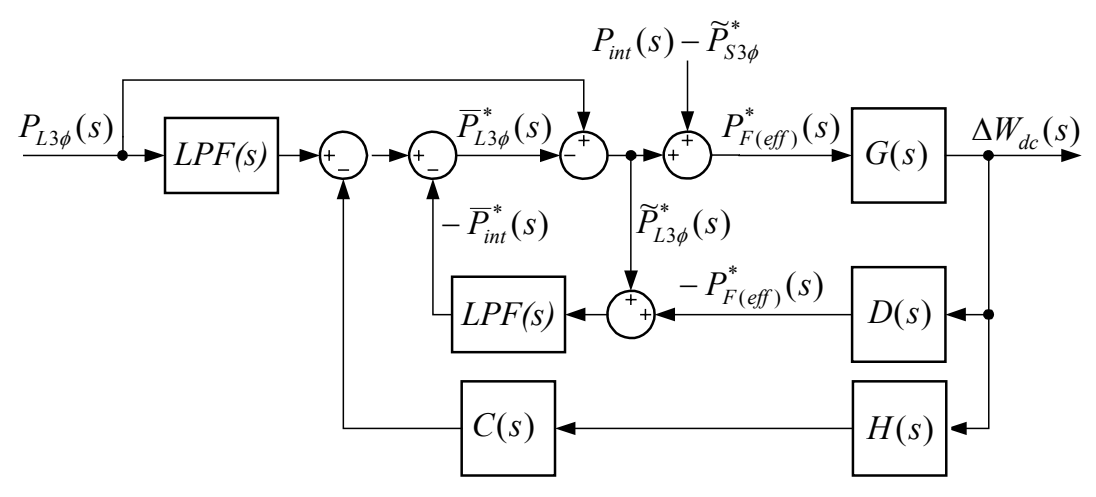

Fig. 6. Control diagram of the dc-bus energy variation controller using a double feedback loop 


$$
\begin{aligned}
& F_{1}(s)=\frac{1}{1-\operatorname{LPF}(s)}=1+\frac{\omega_{f}^{2}}{s\left(s+2 \omega_{f}\right)} \\
& F_{2}(s)=D(s) \frac{\operatorname{LPF}(s)}{1-\operatorname{LPF}(s)}=\frac{\omega_{f}^{2}}{s+2 \omega_{f}}
\end{aligned}
$$

Expression (30) leads to the definitive diagram, shown in Fig. 7, for the SAPF controller based on the dc-bus energy regulation.

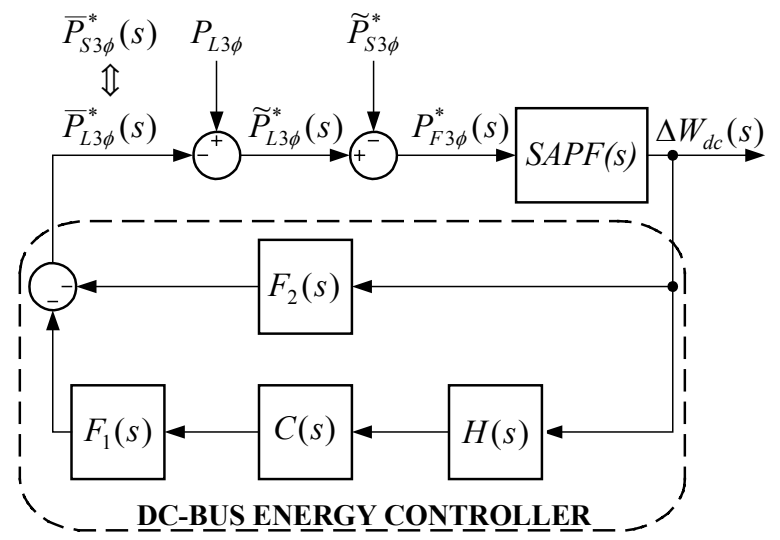

Fig. 7. Definitive SAPF controller diagram

Implementation of this definitive SAPF control diagram is shown in Fig. 7, where the reference current for leg $d$ is obtained by means of $i_{F d}^{*}=-\left(i_{F a}^{*}+i_{F b}^{*}+i_{F c}^{*}\right)$, and power to current transformation is given by:

$i_{S i}^{*}=\left.\left[P \rightarrow I^{+1}\right] \cdot v_{S i}^{+1}\right|_{i=a, b, c} \quad ; \quad\left[P \rightarrow I^{+1}\right]=\frac{\bar{p}_{L 3 \phi}^{*}}{\sum_{j=a, b, c}\left(v_{S j}^{+1}\right)^{2}}$

In Fig. 7, the dc-bus energy variation is calculated by means of (27) from the dc-bus absolute voltage sensing. This is the input variable of the dc-bus energy controller, and its output variable is the mean value of the instantaneous active power that should be supplied by the source. This value of power acts as an input for the power to current transformation. The other input of this transformation is the positive sequence utility voltage at fundamental frequency, which is obtained from the SDRF-PLL. The output of this transformation is the set of purely active sinusoidal currents that should flow from the source side. Subtracting this source reference current from the instantaneous load current, the reference current for the SAPF is obtained.

\section{Simulation results}

As (30) shows, the proposed controller permits to have full control of the dc-bus energy state versus any of the power terms that affect to the SAPF. For this reason, it will be simulated a scene where the SAPF is not only compensating load current harmonics, but also supplying an additional load connected to its dc-bus.

The nonlinear load to be compensated consists in a battery charger connected between phase $b$ and the neutral conductor. This battery charger has a single-phase full-bridge uncontrolled rectifier in the ac side and an inductive filter in the dc side. The initial voltage level of the battery has been fixed in order to achieve that, in steady state, the mean value of the instantaneous active power consumed from the source is equal to $1.5 \mathrm{~kW}$. The additional load connected to the dc-bus consists in a resistive load that also consumes $1.5 \mathrm{~kW}$. In the utility, the rms phase voltage is equal to $220 \mathrm{~V}$ and the frequency is equal to $50 \mathrm{~Hz}$, so that characteristic parameters for the controller are: $k=\omega_{f}=2 \cdot \pi \cdot 10 \mathrm{~W} / \mathrm{J}, \omega_{h}=2 \cdot \pi \cdot 100 \mathrm{rad} / \mathrm{s}$ and $\xi_{f}=\xi_{h}=1$. The value of the dc-bus capacitors is $C_{l}=C_{2}=$ $2000 \mu \mathrm{F}$, and the reference value for the dc-bus absolute voltage is $v_{d c(r e f)}=700 \mathrm{~V}$.

To test the dynamic response of the controller, the resistive load is connected to the dc-bus at $t=5 \mathrm{~ms}$. Later, at $t=0.2 \mathrm{~s}$, the battery charger is connected to the utility. At $t=0.4 \mathrm{~s}$, the battery charge is disconnected from the utility. Finally, at $t=0.6 \mathrm{~s}$, the resistive load is disconnected from the dc-bus. From (25), and taking into account the characteristic parameters for the controller and the converter, each time that one load is connected or disconnected, the maximum absolute value of the dc-bus energy variation should be $\left|\Delta w_{d c(\max )}\right|=17,52 \mathrm{~J}$.

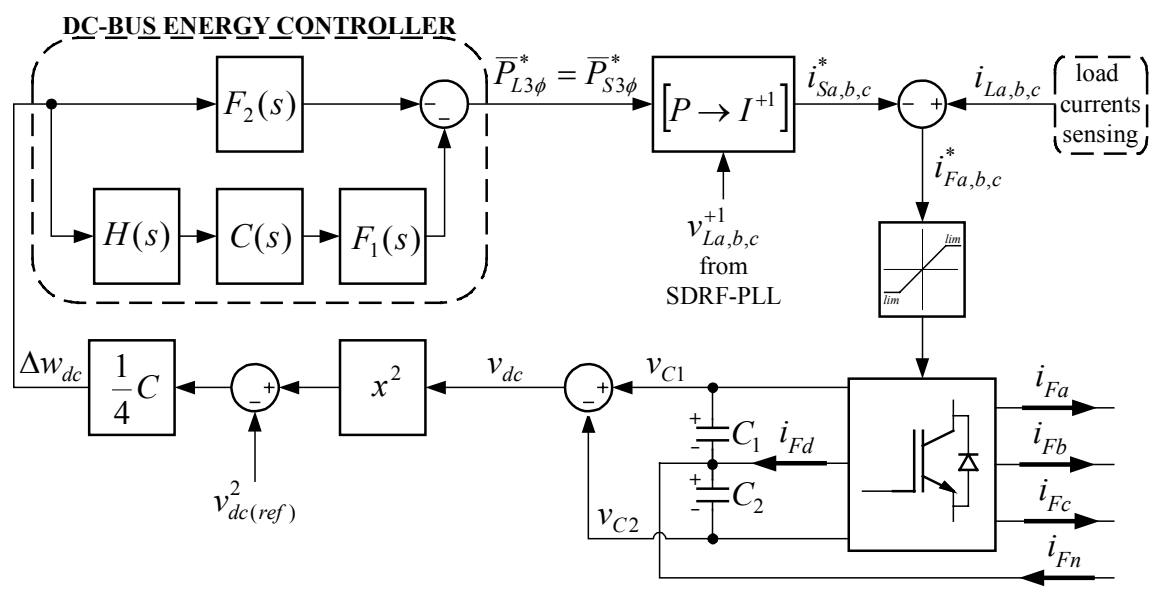

Fig. 8. Implementation of the SAPF controller by means of dc-bus energy regulation 
a)

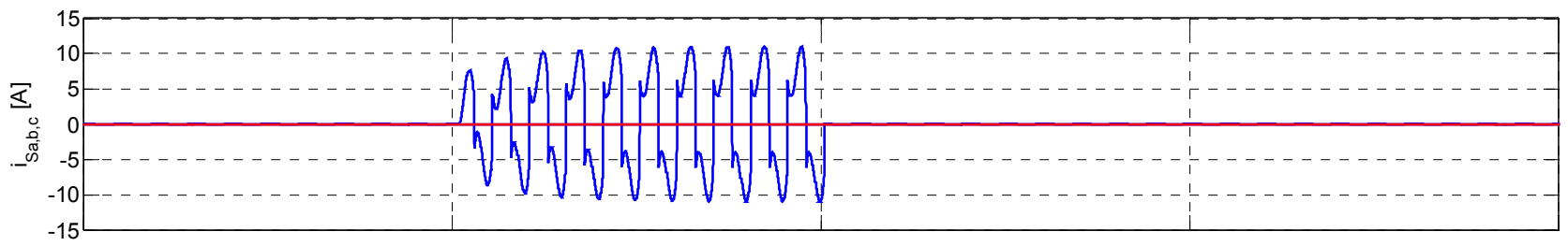

b)

过

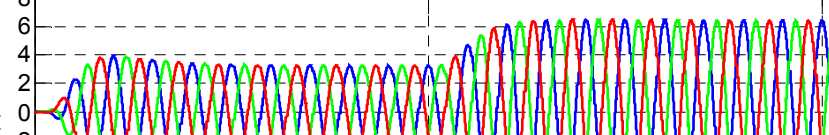

. लึ -2

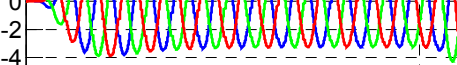

-4
-6

c)

$\Sigma$

d)

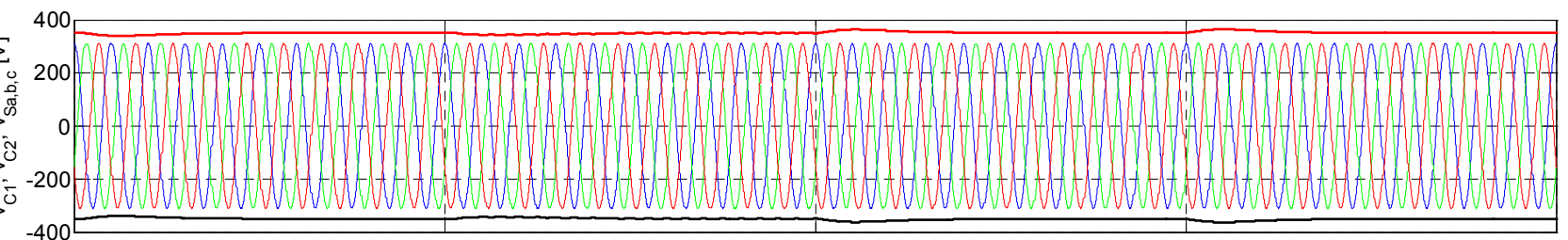

$\sum 2000$

3500

3000

2500

¿ 1000

1000
500
0
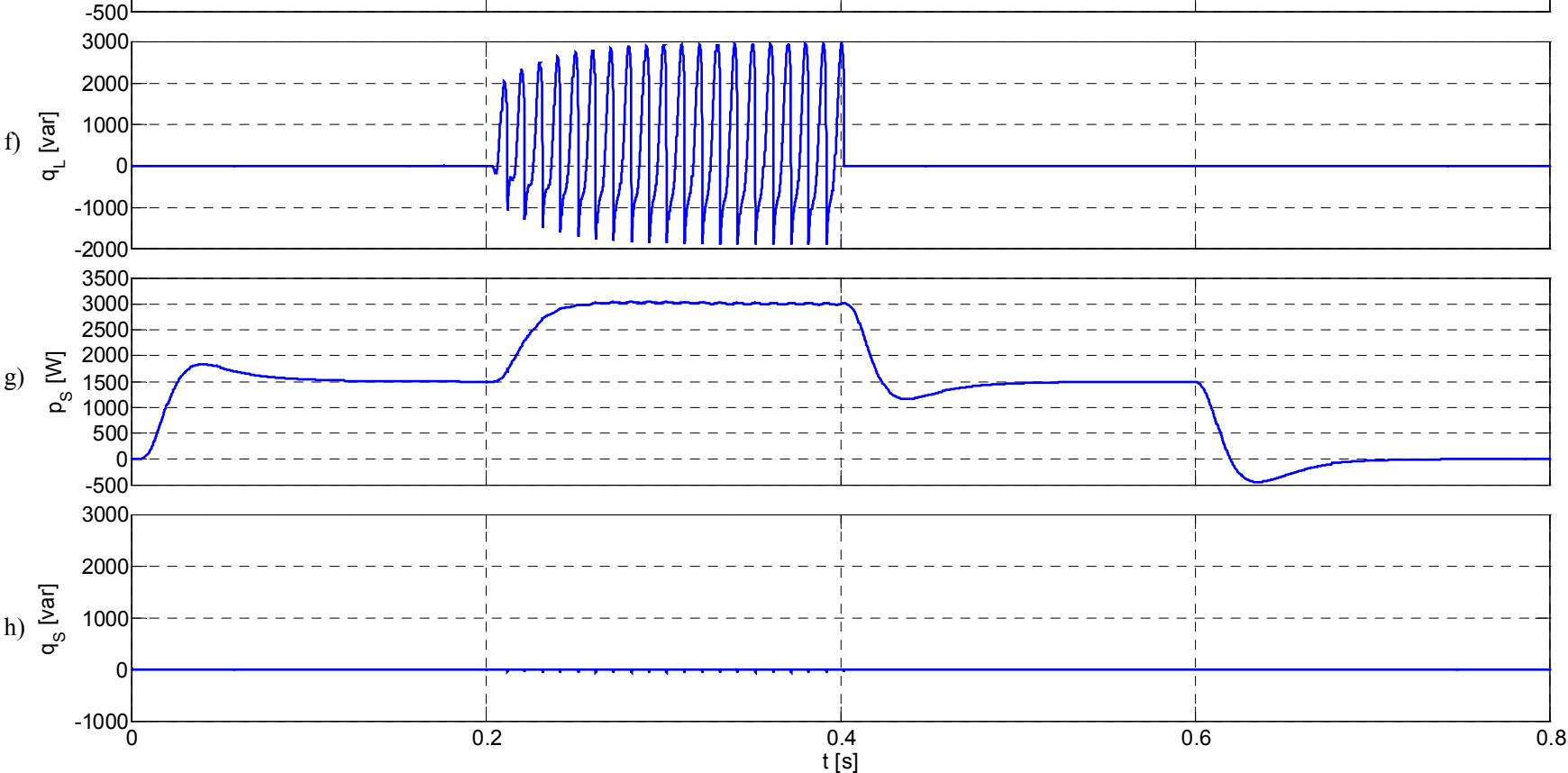

Fig. 9 Simulation results for the proposed SAPF controller. a) load currents, b) source currents, c) dc-bus energy variation, d) Capacitors and utility voltages, e) load instantaneous real power, f) load instantaneous imaginary power, e) source instantaneous real power, and f) source instantaneous imaginary power

In Fig. 9 simulation results are shown. Plot d) of this figure shows dc-bus energy variation. Connection $(t=5 \mathrm{~ms})$ and disconnection $(t=0.6 \mathrm{~s})$ of the resistive load gives rise to almost perfect active power steps, and thereby, in presence of such events, the maximum absolute value of the dc-bus energy variation closely coincides with the theoretically calculated value. Connection of the battery charger $(t=0.2 \mathrm{~s})$, does not 
generate a perfect step in the instantaneous active power consumed by this load because a transitory period is necessary to charge the inductive filter in its dc-side. This effect can be perfectly observed in the plot e), which represents the instantaneous real power consumed by the battery charger. Therefore, the maximum absolute value of the dc-bus energy variation after battery charger connection is different from the theoretic value. After battery charger connection, oscillating components of the load power are being compensated by the SAPF, and hence, energy oscillations appear in the dc-bus. The sudden disconnection of the battery charger $(t=0,4 \mathrm{~s})$ gives rise also to a clean step in the power consumed by the load, and the maximum value of the dc-bus energy variation after of this event coincides again with the theoretical value. The good behavior of the proposed controller can be observed in the plot g), where a freeoscillation real power evolution is shown in spite of the fact that a very undesirable load has been connected.

\section{Conclusion}

From the study presented in this paper, it can be concluded that the proposed controller provides an effective method for reference currents calculation, based on energy state of the SAPF. Analyzing the SAPF from an energy approach, conventional capabilities can be extended, and it is possible to add the active filtering function to the conventional three-phase PWM boost rectifier. With this approach, dc-bus capacitors are rated according to dynamic response of the system; therefore, operation conditions of the SAPF can be assured versus sudden variations in load power.

\section{Acknowledgement}

This work was supported by the Ministerio de Ciencia y Tecnología (MCYT) under the Grant DPI2001-2213 and by the Comissionat Interdepartamental de Recerca $\mathrm{i}$ Innovació Tecnològica (CIRIT) under the Grant DPI2001-2192.

\section{References}

[1] C.A. Quinn and N. Mohan, "Active Filtering Currents in Three-Phase, Four-Wire Systems with Three-Phase and Single-Phase Non-Linear Loads," in Proc. IEEE-APEC, pp. 829-836, Feb. 1992.

[2] S. Buso, L. Malesani, and P. Mattavelli, "Comparison of Current Control Techniques for Active filter Applications," IEEE Trans. Industrial Electronics, Vol. 45, No. 5, pp. 722-729, Oct. 1998.

[3] P. Verdelho and G.D. Marques, "Four-Wire CurrentRegulated PWM Voltage Converter," IEEE Trans. Industrial Electronics, Vol. 45, No. 5, pp. 761-770, Oct. 1998.

[4] R. Zhang, V.H. Prasad, D. Boroyevich and F.C. Lee, "Three-Dimensional Space Vector Modulation for Four-Leg Voltage-Source Converters," IEEE Trans. Power Electronics, Vol. 17, No. 3, pp. 314-326, May 2002.

[5] P. Rodríguez, R. Pindado and J. Bergas, “Alternative Topology for Three-Phase Four-Wire PWM Converters Applied to a Shunt Active Power Filter," in Proc. IEEE-IECON, Vol. 4, pp. 2939-2944, Nov. 2002.

[6] P. Rodríguez, R. Pindado and J. Pou, "Energy Control of Three-phase Four-wire Shunt Active Power Filter," in Proc. IEEE-IECON, pp. 10611066, Nov. 2003.

[7] P. Rodríguez, J. Bergas, and L. Sainz, "New PLL Approach Considering Unbalanced Line Voltage Condition," in Proc. IEEE-EuroPES, pp. 329-334, June 2002.

[8] P. Rodríguez, L. Sainz and J. Bergas, "Synchronous Double Reference Frame PLL applied to Unified Power Quality Conditioner," in Proc. IEEE-ICHQP, pp. 614-619, Oct. 2002.

[9] Q.-C. Zhong, T.C. Green, J. Liang and G. Weiss, " $\mathrm{H}^{\infty}$ Control of the Neutral Point in 3-phase 4-wire DC-AC Converters," in Proc. IEEE-IECON, Vol. 1, pp. 520-525, Nov. 2002.

[10]M.K. Mishra, A. Joshi and A. Ghosh, "Control Schemes for Equalization of Capacitor Voltages in Neutral Clamped Shunt Compensator," IEEE Trans. Power Delivery, Vol. 18, No. 2, pp. 538-544, April 2003. 